\title{
Visceral, general, abdominal adiposity and atherogenic index of plasma in relatively lean hemodialysis patients
}

\author{
Chaomin Zhou ${ }^{1 \dagger}$, Hongying Peng ${ }^{2 \dagger}$, Jing Yuan ${ }^{1}$, Xin Lin ${ }^{1}$, Yan Zha ${ }^{1 *}$ and Hui Chen ${ }^{3 *}$
}

\begin{abstract}
Background: Obesity is a well-established risk factor for atherosclerosis. However, it is unknown which measure of adiposity best relates to atherosclerosis in relatively lean maintenance hemodialysis (MHD) patients. We aimed to explore and compare the associations between different adiposity indices reflecting general, abdominal, visceral adiposity and arteriosclerosis risk with atherogenic index of plasma(AIP) in relatively lean MHD patients.

Methods: We conducted a multicenter, cross-sectional study in Guizhou Province, Southwest China. General/ abdominal adiposity indices like body mass index (BMI), waist circumference(WC), waist-height ratio(WHtR), conicity index (Ci) and visceral obesity indices including visceral adiposity index (VAl), lipid accumulation product (LAP) and the hypertriglyceridemic waist phenotype (HW phenotype) were recorded. Univariate and multivariate linear regression models were used.
\end{abstract}

Results: All adiposity indices correlated positively with AIP in univariate analysis both in men and women except for Ci. After adjustment for age and traditional atherosclerosis risk factors, BMI, WC, WHtR, VAl and LAP still had associations with AIP both in men $(\beta=0.265,0.153,0.16,0.788$ and 0.74 , respectively, all $P<0.001)$ and women ( $\beta=0.34,0.199,0.21,0.83$ and 0.74 , respectively, all $P<0.001$ ). After further adjustment for $B M l$, associations between AIP and VAI, LAP remained significant, but associations between WC, WHtR and AIP disappeared.

Conclusions: The HW phenotype, VAl, and LAP, validated and convenient markers of visceral obesity, were superior to classical anthropometric general/ abdominal adiposity indices for atherosclerosis risk assessment, especially in relatively lean MHD patients aged 40 years or older.

Keywords: Visceral adiposity, Hemodialysis, Atherogenic index of plasma

\section{Background}

Cardiovascular diseases (CVD) still represent the leading cause of mortality in maintenance hemodialysis (MHD) patients [1] and atherosclerosis is thought to be a primary contributor to CVD. It is generally acknowledged that end-stage renal disease (ESRD) patients suffer from accelerated atherosclerosis. Diffuse atherosclerosis may be observed even in early stages of chronic kidney

\footnotetext{
*Correspondence: zhayan72@126.com; xtd-111@163.com

${ }^{+}$Chaomin Zhou and Hongying Peng contributed equally to this work. ${ }^{1}$ Renal Division, Department of Medicine, Guizhou Provincial People's Hospital, Guizhou Provincial Institute of Nephritic and Urinary Disease, Guiyang 550002, Guizhou, China

${ }^{3}$ The ministry of science and education, Guizhou Provincial People's Hospital, Guiyang 550002, Guizhou, China

Full list of author information is available at the end of the article
}

disease (CKD) [2]. Early identification and intervention for atherosclerosis risk is therefore critical.

Adiposity is a well-known precursor of atherosclerosis. Adiposity could be widely divided into general adiposity which is usually determined by body mass index (BMI) and central adiposity with waist circumference (WC) as the most common measure. Though BMI is a confirmed indicator of obesity, fluid overload should not be overlooked, especially in MHD patients. Besides, BMI does not differentiate the types of adipose tissues as well as the body fat distribution. Though markers of central adiposity such as WC, waist height ratio (WHtR) and conicity index $(\mathrm{Ci})$ provide a better estimation to evaluate proportion of body fat and fat distribution than BMI [3], they are incapable of differentiating subcutaneous fat

(C) The Author(s). 2018 Open Access This article is distributed under the terms of the Creative Commons Attribution 4.0 International License (http://creativecommons.org/licenses/by/4.0/), which permits unrestricted use, distribution, and 
and visceral fat, which play opposite roles in the development of atherosclerosis [4]. The importance of visceral adiposity has received increasing attention this decade $[5,6]$. Many studies have indicated that it is visceral obesity rather than subcutaneous obesity that relate to metabolic abnormalities [7, 8]. Visceral adiposity index(VAI), the hypertriglyceridemic waist phenotype(HW phenotype)and lipid accumulation product (LAP) are validated and convenient markers of visceral obesity. They are reported to be associated with subclinical atherosclerosis and intracranial atherosclerotic stenosis $[6,9]$. Chinese are more likely to be viscerally obesity or centrally obesity in spite of having generally low BMI $[10,11]$. However, it is unknown which measure of adiposity best relates with atherosclerosis in relatively lean MHD patients.

Atherogenic index of plasma (AIP) is the logarithm of the plasma triglyceride(TG) level to high-density lipoprotein cholesterol (HDL-c) (log [TG/HDL-c])ratio [12], and is thought to be a good marker of atherogenicity and can be used in the diagnosis of subclinical atherosclerosis [13]. In the present study, we investigated and compared the sex-specific associations between different adiposity indices reflecting general adiposity(BMI), abdominal adiposity (WC, WHtR and Ci), visceral adiposity (VAI, HW phenotype, LAP) and arteriosclerosis risk with AIP in relatively lean MHD patients.

\section{Methods}

\section{Participants}

The Ethics Committee of The People's Hospital of Guizhou province approved this study. This study was performed fulfilling the principles of Helsinki Declaration. We conducted a multicenter, cross-sectional study in the hemodialysis centers of 11 hospitals in Guizhou Province, Southwest China from June 1, 2016 to September 30, 2016. All MHD patients were invited to participate in our study and all participants received 4-h carbonate-based dialysis treatment twice or thrice weekly. Adult patients on standard MHD at these 11 hemodialysis centers for at least 3 months or longer were included in our study. Subjects with hearing disabilities, language barriers, mental disability, and any physical deformities, comorbid with cancer, treated with lipid-lowering drugs were excluded from our study. We also excluded subjects on any kind of nutritional support and patients with obvious edema or ascites. All participants were voluntary to take part in our study and had submitted their written consent. As $\mathrm{BMI}<25 \mathrm{~kg} / \mathrm{m}^{2}$ was considered non-obese according to ranges established for Asian populations [14], only adult patients with $\mathrm{BMI}<25 \mathrm{~kg} / \mathrm{m}^{2}$ were included in the final analysis.

\section{Data collection}

A predesigned questionnaire was used to conduct a standardized, structured interview. The questionnaire consisted of demographic and socioeconomic information such as age, gender, personal history (coronary artery disease, hypertension, and diabetes) and details about lifestyle of the participants. Laboratory parameters including TG, cholesterol (CHOL), HDL, low-density lipoprotein-cholesterol (LDL) et al. were extracted from the medical records by researchers. Participants who did not have laboratory parameters in the past 3 months were excluded in the final analysis.

\section{Anthropometric measures}

Anthropometric measurements were conducted in the dialysis centers after dialysis session by well-trained nurses and physicians. Weight was assessed using an electronic scale with the patients in light clothes and without shoes. Participants were asked to stand with arms hanging freely and height was acquired by a stadiometer with patient barefoot. BMI was calculated as weight $(\mathrm{kg})$ divided by height squared $\left(\mathrm{m}^{2}\right)$. WC was measured using an inelastic tape measure. Participants were asked to wear light clothes and WC was assessed midway between the last rib and iliac crest with the participants breathing out gently. Duplicate measures were taken for all measurements with a tolerance error of $1 \mathrm{~cm}$ for height and circumference measurements, and $1 \mathrm{~kg}$ for the weight measurement. A third measurement was needed if the difference of the first two measures was greater than tolerance limit. The average of the two closest measurements was recorded at last. WHtR was calculated according to the following formula: WC $(\mathrm{cm})$ /height $(\mathrm{cm})$ [15]. CI was calculated according to the formula $[16,17]:$ WC $(\mathrm{m}) /[0.109 \times$ square root of weight (kg) /height (m)]. LAP index was calculated according to the published formula [18]: (WC $(\mathrm{cm})-65) \times \mathrm{TG}$ $(\mathrm{mmol} / \mathrm{l})$ for men and $(\mathrm{WC}(\mathrm{cm})-58) \times \mathrm{TG}(\mathrm{mmol} / \mathrm{l})$ for women.

\section{Definition of VAI score}

The VAI score was derived according to a published formula [19]:

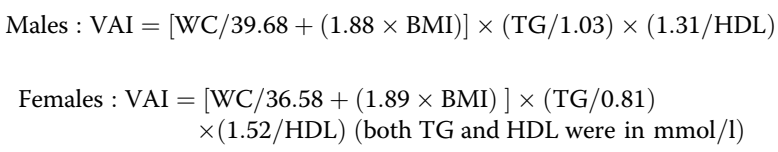

\section{Definition of the HW phenotype}

The HW phenotype was defined as elevated WC $(>90 \mathrm{~cm}$ in men and $>80 \mathrm{~cm}$ in women), along with an elevated plasma TG concentration $\geq 1.7 \mathrm{mmol} / \mathrm{l}$ for both genders [20]. As the HW phenotype was proposed to be a 
surrogate marker of visceral obesity, subjects were divided into 3 groups by the presence of the HW components. Group 1, normal WC (WC $<90 \mathrm{~cm}$ in men or $<80 \mathrm{~cm}$ in women) and normal TG level (TG $<1.7 \mathrm{mmol} / \mathrm{L})$ for both genders; Group 2, solely WC increase (WC $\geq 90 \mathrm{~cm}$ in men or $\geq 80 \mathrm{~cm}$ in women, along with $\mathrm{TG}<1.7 \mathrm{mmol} / \mathrm{l}$ ) or solely TG increase (WC $<90 \mathrm{~cm}$ in men or $<80 \mathrm{~cm}$ in women along with TG $\geq 1.7 \mathrm{mmol} / \mathrm{l}$ ); Group 3, increased WC and TG level $(W C \geq 90 \mathrm{~cm}$ in men or $\geq 80 \mathrm{~cm}$ in women, and $\mathrm{TG} \geq 1.7 \mathrm{mmol} / \mathrm{l})$.

\section{Statistical analysis}

Statistical analyses were conducted using SPSS software (version 13.0 for windows; SPSS, Chicago, IL, USA). Continuous variables were presented as median and interquartile range due to their skewed distribution. Mann-Whitney $U$ test was used to compare continuous variables in male and female participants. Categorical variables were expressed as percentages. The chi-squared test was used to assess differences in proportions between groups for categorical variables. Spearman correlation analysis was used to evaluate the association between the HW phenotype and AIP. Univariate and multivariate linear regression models were used to examine the associations between various markers of obesity (BMI, WC, WHtR, Ci, VAI and LAP) and AIP. A two-tailed $P$ value less than 0.05 was considered statistically significant.

\section{Results}

Initially there were 939 participants included in our study, 30 participants were excluded from our final analysis because of missing data for TG, CHOL, HDL and LDL. The main anthropometric and clinical characteristics of the study population were presented in Table 1 . As shown in Table 1, levels of TG, HDL, LDL and CHOL in women were higher as compared to men. Men exhibited increased BMI and WC than women $(P<0.05)$. There was no statistical difference in AIP between male and female participants. More than $80 \%$ of the participants were aged 40 years and older. $21.7 \%$ women had the HW phenotype, while only $8.8 \%$ men had this phenotype. VAI and LAP were higher in women as compared to men $(P<0.001)$.

\section{Relationship between various measures of general/central obesity and AIP}

Univariate correlations between various measures of general/central obesity and AIP were shown in Fig. 1. Ci had no significant correlation with $\operatorname{AIP}(P>0.05)$. BMI, WC and WHtR were positively associated with AIP in the entire population $(r=0.26,0.17,0.169$, respectively, all $P<0.001)$. As shown in Fig. 1, BMI showed stronger correlation with AIP $(r=0.24$ for men, $r=0.29$ for women, $\mathrm{P}<0.001)$ than WC $(r=0.15$ for men, $r=0.21$ for women, $\mathrm{P}<0.001)$ and WHtR $(r=0.144$ for men and $\mathrm{r}=0.21$ for women, $\mathrm{P}<0.001)$ in both men and women. As shown in Table 2, BMI, WC, WHtR still correlated with AIP after adjustment for age and traditional atherosclerosis risk factors such as history of smoking, history

Table 1 Characteristics of the study population stratified by sex

\begin{tabular}{|c|c|c|c|}
\hline Characteristic & Men $(n=551)$ & Women $(n=358)$ & $P$ value \\
\hline Age (years) & $56(43,67)$ & $57(44,70)$ & NS \\
\hline Age $\geq 40$ years $(\%)$ & 80.5 & 80.1 & NS \\
\hline History of smoking (\%) & 55.5 & 6.1 & $<0.001$ \\
\hline History of high blood pressure (\%) & 83.2 & 80.6 & NS \\
\hline History of diabetes N(\%) & 199(36\%) & 113(31\%) & NS \\
\hline Waist circumference $(\mathrm{cm})$ & $82.7(76.25,88.6)$ & $79(73.7,85.13)$ & $<0.001$ \\
\hline Body mass index & $21.45(19.59,23)$ & $20.81(18.94,22.65)$ & $<0.001$ \\
\hline Waist-to-Height Ratio & $0.50(0.46,0.54)$ & $0.51(0.47,0.55)$ & $<0.001$ \\
\hline Conicity index & $1.27(1.21,1.33)$ & $1.29(1.21,1.35)$ & NS \\
\hline Visceral adiposity index & $1.56(0.94,2.61)$ & $2.32(1.43,3.72)$ & $<0.001$ \\
\hline Lipid accumulation product & $21.28(12,37.34)$ & $31.92(17.6,52.5)$ & $<0.001$ \\
\hline Atherogenic index of plasma & $0.07(-0.14,0.29)$ & $0.07(-0.13,0.29)$ & NS \\
\hline Hypertriglyceridemic waist, \% & 8.8 & 21.7 & $<0.001$ \\
\hline Triglycerides (mmol/L) & $1.27(0.93,1.86)$ & $1.46(1.09,2.2)$ & $<0.001$ \\
\hline High density lipoprotein (mmol/L) & $1.08(0.88,1.3)$ & $1.25(1.01,1.52)$ & $<0.001$ \\
\hline Low density lipoprotein (mmol/L) & $2.17(1.7,2.72)$ & $2.34(1.78,3.01)$ & 0.001 \\
\hline Total cholesterol (mmol/L) & $3.78(3.17,4.35)$ & $4.24(3.65,5.03)$ & $<0.001$ \\
\hline
\end{tabular}

Data are presented as $n(\%)$ or median and interquartile range. NS, non- significant $(P>0.05)$ 


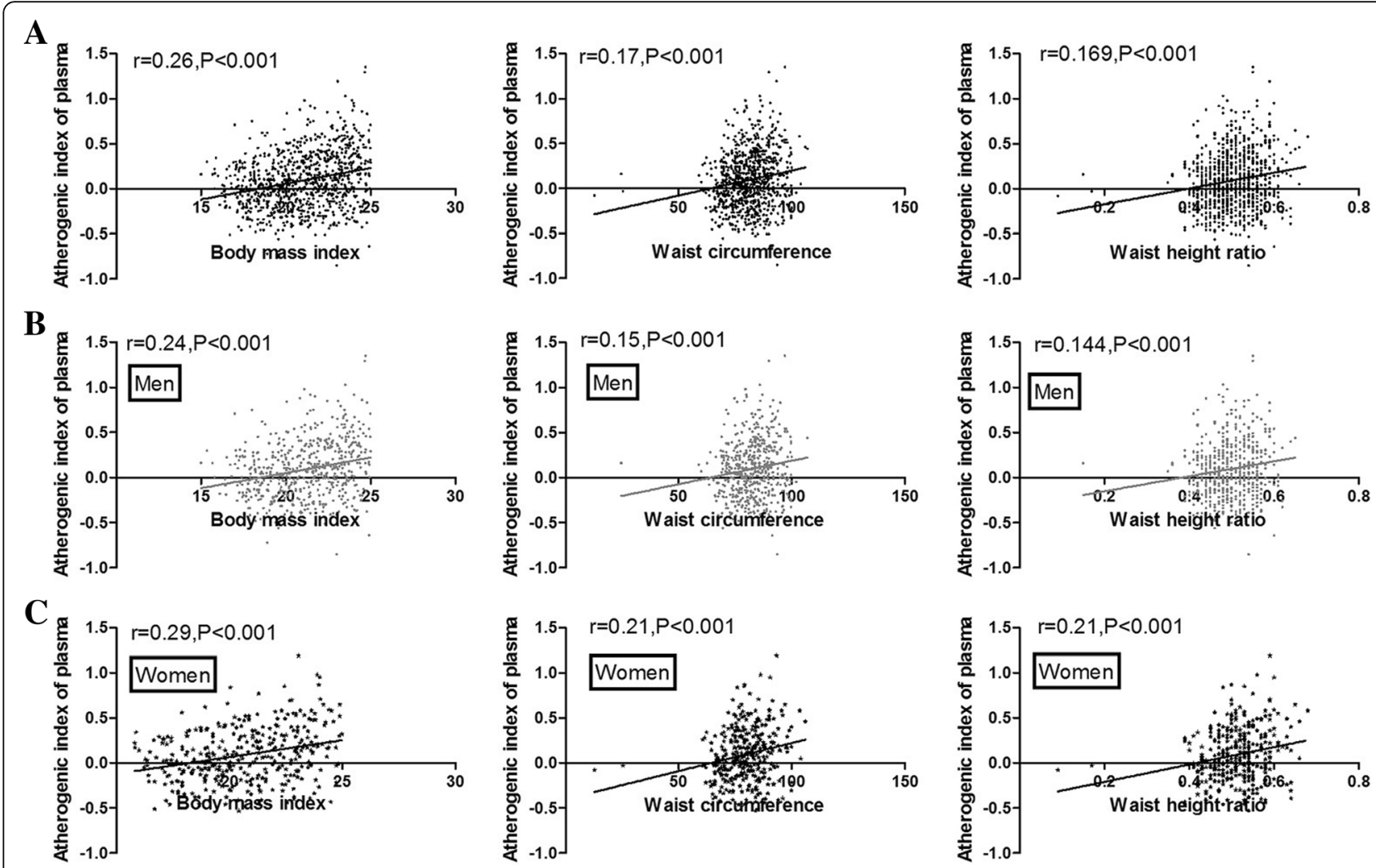

Fig. 1 Relationship between body mass index (BMI), waist circumference(WC), waist-height ratio(WHtR) and atherogenic index of plasma (AIP). a entire subjects; b Men; c women

Table 2 Comparison of the association of various measures of adiposity with AIP after adjusting for traditional atherosclerosis risk factors and BMI

\begin{tabular}{|c|c|c|c|c|}
\hline & \multicolumn{2}{|c|}{ Model 1} & \multicolumn{2}{|c|}{ Model 2} \\
\hline & $\bar{\beta}$ & $P$ value & $\beta$ & $P$ value \\
\hline \multicolumn{5}{|l|}{ Men } \\
\hline BMI & 0.265 & $<0.001$ & & \\
\hline WC & 0.153 & 0.001 & NS & NS \\
\hline $\mathrm{WH} t \mathrm{R}$ & 0.16 & 0.001 & NS & NS \\
\hline VAl & 0.788 & $<0.001$ & 0.77 & $<0.001$ \\
\hline LAP & 0.74 & $<0.001$ & 0.725 & $<0.001$ \\
\hline \multicolumn{5}{|l|}{ Women } \\
\hline BMI & 0.34 & $<0.001$ & & \\
\hline WC & 0.199 & 0.001 & NS & \\
\hline $\mathrm{WH}$ tR & 0.21 & $<0.001$ & NS & \\
\hline VAl & 0.83 & $<0.001$ & 0.816 & $<0.001$ \\
\hline LAP & 0.74 & $<0.001$ & 0.73 & $<0.001$ \\
\hline
\end{tabular}

Model 1: adjusted for age, history of smoking,history of high blood pressure and history of diabetes;

Model 2: adjusted for above + BMI

BMI, body mass index; WC, waist circumference; WHtR, waist height ratio; VAl, visceral adiposity index; LAP, lipid accumulation product

NS, non- significant $(P>0.05)$ of high blood pressure and history of diabetes both in men and women. However, the associations disappeared after further adjustment for $\operatorname{BMI}(P>0.05)$.

Relationship between markers of visceral obesity and AIP As shown in Table 3, there was a moderate correlation between HW phenotype and AIP $(r=0.601$ for men, $r=$ 0.693 for women, $p<0.0001)$. VAI and LAP were significantly and positively correlated with AIP $\left(\mathrm{r}_{\mathrm{VAI}}=0.802\right.$, $r_{\mathrm{LAP}}=0.71$ for men, $\mathrm{r}_{\mathrm{VAI}}=0.84, \mathrm{r}_{\mathrm{LAP}}=0.72$ for women, $\mathrm{p}$ $<0.0001)$. VAI showed the strongest correlation with AIP $(\beta=0.8, P<0.001)$ in overall subjects. After adjustment for age and traditional atherosclerosis risk factors (i.e. history of smoking,history of high blood pressure and history of diabetes), VAI and LAP still had strong associations with AIP in men $(\beta=0.788$ and 0.74 , respectively, both $\mathrm{P}<0.001)$ and women $(\beta=0.83$ and 0.74 , respectively, both $\mathrm{P}<0.001$ ) as shown in Table 3 . These associations remained, although slightly attenuated, after further adjustment for BMI (all $\mathrm{P}<0.001$ ).

\section{Discussion}

Adiposity is a known major determinant of atherosclerosis in the general population. Whether adiposity indexes are associated with atherosclerosis in relatively lean MHD 
Table 3 Pearson / Spearman correlation coefficients for visceral obesity markers and AIP

\begin{tabular}{llll}
\hline & Visceral adiposity index & Hypertriglyceridemic waist phenotype & Lipid accumulation product \\
\hline $\begin{array}{l}\text { Overall }(n=909) \\
\text { Standardized coefficients } \beta\end{array}$ & 0.8 & & 0.70 \\
$P$ & $<0.001$ & $<0.044$ & $<0.001$ \\
Male $(n=551)$ & & & 0.71 \\
Standardized coefficients $\beta$ & 0.802 & 0.601 & $<0.001$ \\
$P$ & $<0.001$ & $<0.001$ & 0.72 \\
Female $(n=358)$ & & & $<0.001$ \\
Standardized coefficients $\beta$ & 0.84 & 0.693 & $<0.001$ \\
$P$ & $<0.001$ & &
\end{tabular}

patients is unknown. Moreover, there are few studies to compare the relative strength of the associations between obesity indexes reflecting general, abdominal, visceral adiposity and atherosclerosis risk. To the best of our knowledge, this is the first study investigating and comparing associations between general, abdominal and visceral adiposity, estimated by obesity indexes and atherosclerosis risk with AIP in relatively lean MHD patients. We found that increase of anthropometric obesity indicators, especially indicators reflecting visceral adiposity was positively associated with atherosclerosis risk even in relatively lean MHD patients. Surrogate markers of visceral obesity were superior to traditional anthropometric obesity indicators, reflecting general and abdominal obesity in identifying atherosclerosis risk. Moreover, associations between markers of visceral obesity and AIP were independent of BMI and traditional atherosclerosis risk factors in relatively lean MHD patients.

The AIP has been suggested to be a good marker of atherogenicity [13]. It was found to be associated with high cardiovascular risk and adverse clinical outcomes $[12,21]$. In the present study, BMI showed the strongest correlation with AIP in entire population among various measures of general/central obesity (BMI, WC, WHtR and $\mathrm{Ci}$ ). While Brodsky demonstrated an inverse relationship between obesity, generally determined by BMI, and subsequent aortic atherosclerosis, which has been termed the "obesity paradox" [22]. In fact, "obesity paradox" exists in diverse diseases including ESRD [23]. "Obesity Paradox" is still controversial [24] and several studies propose that the use of BMI, which is a poor indicator of body fat distribution, as a determinant of obesity may be part of the explanation for "Obesity Paradox" [25]. In addition, fluid overload should be considered especially in ESRD patients. Central obesity is thought to be more pathogenic and more important as a predictor of cardiovascular metabolic disease compared to general obesity [26, 27]. General obesity as assessed by BMI, however, showed a stronger relation with AIP than central obesity as assessed by WC, WHtR in our study. Antoninus et al. [28] demonstrated that a significant correlation was obtained between each of the anthropometric measures and AIP in non-obese sedentary men except for $\mathrm{Ci}$. Consistent with the findings in Antoninus's study, Ci showed no association with AIP in our study. In their study, BMI had a stronger correlation with AIP compared with other obesity indicators such as WC, WHtR, WHR, which was similar to our results. Though WC, WHtR correlated with AIP, the associations were weak and depend on BMI in our study. Actually, these results were in compliance with previous studies which suggested that BMI was a more powerful predictor of body fat percent than $\mathrm{WC}$ and $\mathrm{Ci}$ [29] and BMI was a stronger predictor of metabolic syndrome (MetS) than WC and Ci [30]. As we all know MetS showed significant relationship with carotid atherosclerosis [31].

Though central obesity indicators such as WC and WHtR were recommended to be relatively ideal markers of obesity [32]. It is rather difficult for these markers to identify subcutaneous and visceral adiposity. The importance of visceral obesity has been emphasized by a lot of studies [5, 6]. Studies have indicated that males and postmenopausal females tend to deposit more visceral fat [33]. More than $80 \%$ patients were above 40 years in the present study. Most women reach menopause during age 45 to 55 years, but it may occur from age 40 to 60 years [34]. We could speculate therefore, most of our female patients were menopausal. This might be a reasonable explanation for women were more likely to have visceral obesity in the present study, as more women had the HW phenotype and LAP, VAI were much higher in women compared to men. Our previous studies and other studies from the Chinese population had come to similar conclusions [20, 35, 36]. We found strong positive associations between visceral obesity indicators (VAI, LAP and HW phenotype) and atherosclerosis risk both in men and women. VAI and LAP seemed to have a stronger relation with AIP in women compared to men. These associations were independent of BMI and traditional atherosclerosis risk factors 
such as age, history of smoking,history of high blood pressure and history of diabetes. Our results might partially contribute to the middle-aged population as more than $80 \%$ subjects were aged 40 or older, and this group was particularly exposed to increased risk of developing the cardiometabolic profile. Our results were supported by previous studies which suggested that TG/HDL-C concentration was highly correlated with VAI $[19,37]$. Another study has reached a similar conclusion [38]. A recent study has suggested that visceral fat mass, estimated by Dual-energy X-ray absorptiometry (DXA) was negatively associated with HDL cholesterol and positively associated with TG [39]. As we know AIP is logarithmic ratio of TG/ HDL-C. Data also suggest that VAI is positively correlated with coronary artery calcium score (CACS), a marker of subclinical atherosclerosis [40] and the association is still significant even after adjusting for confounding variables, including BMI. Previous studies have indicated that the HW phenotype is highly associated with CACS [41] and LAP outperforms BMI in identifying metabolic risk factors [42] and predicting all-cause mortality [43]. A previous study suggested that accumulated abdominal visceral fat was associated with artery intima-media thickness [44].

Exact mechanisms related to the associations between markers of visceral obesity and atherosclerosis risk in relatively lean MHD patients are yet to be clearly established. Data suggest that visceral fat depots and hypertrophic adipocytes drive the secretion of proinflammatory cytokines such as interleukine-6 (IL-6), tumor necrosis factor-alpha(TNF-a). Many of these cytokines have a detrimental effect on atherogenesis, plaque progression and thrombosis [45, 46]. Animal experiments demonstrate that enhanced atherosclerosis can be attributed to a cross-talk between visceral fat and the vasculature, which is mediated by the release of proinflammatory cytokines [47]. But these explanations have not been precisely defined in human and are likely to be multifactorial, further studies are needed.

Limitations in the current study deserve to be illustrated. First, the cross-sectional nature of our study disabled us to make causal inferences between obesity and atherosclerosis in non-obese MHD patients, prospective studies are needed. Second, subjects in this study were all Chinese, extrapolating results to other populations should be interpreted cautiously. Third, there was an age imbalance in our study population, over $80 \%$ participants in our study were aged 40 years and older. Fourth, the sample size was relatively small. However, this is the first study to investigate and compare the sex-specific associations between different adiposity indices reflecting general adiposity, abdominal adiposity, visceral adiposity and arteriosclerosis risk with AIP in relatively lean MHD patients. Therefore, despite these limitations, our study is valuable.

\section{Conclusions}

The HW phenotype, VAI, and LAP, validated and convenient markers of visceral obesity, were superior to classical anthropometric general/ abdominal adiposity indices for atherosclerosis risk assessment, especially in relatively lean MHD patients aged 40 years or older.

\begin{abstract}
Abbreviations
AIP: Atherogenic index of plasma; BMI: Body mass index; Ci: Conicity index; HW phenotype: Hypertriglyceridemic waist phenotype; LAP: Lipid accumulationproduct; MHD: Maintenance hemodialysis; VAl: Visceral adiposity index; WC: Waist circumference; WHtR: Waist-to-height ratio

\section{Acknowledgments}

We thank all participants for their help.

Funding

This study was supported by the following Science Foundation: 1.ZKHT ((20161001)38) from Guiyang City Science and Technology Planning Project; 2. GZSYQN(2016)11.
\end{abstract}

\section{Availability of data and materials}

The datasets used in the study are available from the corresponding author on reasonable request.

\section{Authors' contributions}

Study design: ZCM, ZY, CH; data collection and analysis: PHY, ZCM, LX, YJ; paper draft: ZCM, PHY, ZY; paper revise: ZY, CH. All authors read and approved the final manuscript.

\section{Ethics approval and consent to participate}

Ethics Committee of The People's Hospital of Guizhou province approved the study, with written consent obtained. All participants were voluntary to take part in our study and had submitted their written consent.

Consent for publication

Not applicable.

Competing interests

The authors declare that they have no competing interests.

\section{Publisher's Note}

Springer Nature remains neutral with regard to jurisdictional claims in published maps and institutional affiliations.

\section{Author details}

${ }^{1}$ Renal Division, Department of Medicine, Guizhou Provincial People's Hospital, Guizhou Provincial Institute of Nephritic and Urinary Disease, Guiyang 550002, Guizhou, China. ${ }^{2}$ Renal Division, Department of Medicine, Baiyun Hospital Affiliated to Guizhou Medical University, Guiyang 550002, Guizhou, China. ${ }^{3}$ The ministry of science and education, Guizhou Provincial People's Hospital, Guiyang 550002, Guizhou, China.

Received: 25 February 2018 Accepted: 31 July 2018

Published online: 16 August 2018

\section{References}

1. Khan A, Khan AH, Adnan AS, Syed Sulaiman SA, Gan SH, Khan I. Management of Patient Care in hemodialysis while focusing on cardiovascular disease events and the atypical role of hyper-and/or hypotension: a systematic review. Biomed Res Int. 2016:9710965(10):19.

2. Nakayama M, Metoki H, Terawaki H, Ohkubo T, Kikuya M, Sato T, et al. Kidney dysfunction as a risk factor for first symptomatic stroke events in a general Japanese population--the Ohasama study. Nephrol Dial Transplant. 2007;22(7):1910-5.

3. Goyal A, Nimmakayala KR, Zonszein J. Is there a paradox in obesity? Cardiol Rev. 2014;22(4):163-70.

4. Bouchi R, Takeuchi T, Akihisa M, Ohara N, Nakano Y, Nishitani R, et al. High visceral fat with low subcutaneous fat accumulation as a determinant of 
atherosclerosis in patients with type 2 diabetes. Cardiovasc Diabetol. 2015; 14(136):015-0302.

5. Chen YC, Lai SH, Tsai YW, Chang SS. Visceral adiposity index as a predictor of chronic kidney disease in a relatively healthy population in Taiwan. J Ren Nutr. 2017;14(17):30173-5.

6. Li R, Li Q, Cui M, Ying Z, Li L, Zhong T, et al. Visceral adiposity index, lipid accumulation product and intracranial atherosclerotic stenosis in middleaged and elderly Chinese. Sci Rep. 2017;7(1):7951.

7. Keum N, Lee DH, Kim R, Greenwood DC, Giovannucci EL. Visceral adiposity and colorectal adenomas: dose-response meta-analysis of observational studies. Ann Oncol. 2015;26(6):1101-9.

8. Ross R, Aru J, Freeman J, Hudson R, Janssen I: Abdominal adiposity and insulin resistance in obese men. Am J Physiol Endocrinol Metab. 2002; 282(3):

9. Poirier J, Kubow S, Noel M, Dupont C, Egeland GM. The hypertriglyceridemic-waist phenotype is associated with the Framingham risk score and subclinical atherosclerosis in Canadian Cree. Nutr Metab Cardiovasc Dis. 2015;25(11):1050-5.

10. Lear SA, Humphries KH, Kohli S, Chockalingam A, Frohlich JJ, Birmingham $\mathrm{CL}$. Visceral adipose tissue accumulation differs according to ethnic background: results of the multicultural community health assessment trial (M-CHAT). Am J Clin Nutr. 2007;86(2):353-9.

11. Zhang P, Wang R, Gao C, Jiang L, Lv X, Song Y, et al.: Prevalence of Central Obesity among Adults with Normal BMI and Its Association with Metabolic Diseases in Northeast China. PLoS One. 2016; 11(7):

12. Dobiasova M, Frohlich J. The plasma parameter log (TG/HDL-C) as an atherogenic index: correlation with lipoprotein particle size and esterification rate in apoB-lipoprotein-depleted plasma (FER(HDL)). Clin Biochem. 2001;34(7):583-8.

13. Yildiz G, Duman A, Aydin H, Yilmaz A, Hur E, Magden K, et al. Evaluation of association between atherogenic index of plasma and intima-media thickness of the carotid artery for subclinic atherosclerosis in patients on maintenance hemodialysis. Hemodial Int. 2013;17(3):397-405.

14. Appropriate body-mass index for Asian populations and its implications for policy and intervention strategies. Lancet. 2004; 363(9403): 157-63.

15. Obirikorang C, Obirikorang Y, Acheampong E, Anto EO, Toboh E, Asamoah EA, et al.: Association of Wrist Circumference and Waist-to-Height Ratio with Cardiometabolic Risk Factors among Type II Diabetics in a Ghanaian Population. J Diabetes Res. 2018; 19(1838162):

16. Valdez R. A simple model-based index of abdominal adiposity. J Clin Epidemiol. 1991;44(9):955-6.

17. El Said HW, Mohamed OM, El Said TW, El Serwi AB. Central obesity and risks of cardiovascular events and mortality in prevalent hemodialysis patients. Int Urol Nephrol. 2017;49(7):1251-60.

18. Marcadenti A, Fuchs FD, Moreira LB, Gus M, Fuchs SC. Adiposity phenotypes are associated with type-2 diabetes: LAP index, body adiposity index, and neck circumference. Atherosclerosis. 2017;266:145-50.

19. Amato MC, Giordano C, Galia M, Criscimanna A, Vitabile S, Midiri M, et al. Visceral adiposity index: a reliable indicator of visceral fat function associated with cardiometabolic risk. Diabetes Care. 2010;33(4):920-2.

20. Huang J, Zhou C, Li Y, Zhu S, Liu A, Shao X, et al. Visceral adiposity index, hypertriglyceridemic waist phenotype and chronic kidney disease in a southern Chinese population: a cross-sectional study. Int Urol Nephrol. 2015;47(8):1387-96.

21. Turak O, Afsar B, Ozcan F, Oksuz F, Mendi MA, Yayla C, et al. The role of plasma triglyceride/high-density lipoprotein cholesterol ratio to predict new cardiovascular events in essential hypertensive patients. J Clin Hypertens. 2016;18(8):772-7.

22. Brodsky SV, Barth RF, Mo X, Yildiz V, Allenby P, Ivanov I, et al. An obesity paradox: an inverse correlation between body mass index and atherosclerosis of the aorta. Cardiovasc Pathol. 2016:25(6):515-20.

23. Madero M, Katz R, Murphy R, Newman A, Patel K, Ix J, et al. Comparison between different measures of body fat with kidney function decline and incident CKD. Clin J Am Soc Nephrol. 2017;12(6):893-903.

24. Bosello O, Donataccio MP, Cuzzolaro M. Obesity or obesities? Controversies on the association between body mass index and premature mortality. Eat Weight Disord. 2016;21(2):165-74

25. Sakao Y, Ojima T, Yasuda H, Hashimoto S, Hasegawa T, Iseki K, et al.: Serum Creatinine Modifies Associations between Body Mass Index and Mortality and Morbidity in Prevalent Hemodialysis Patients. PLoS One. 2016; 11(3):

26. Li G, Chen X, Jang Y, Wang J, Xing X, Yang W, et al. Obesity, coronary heart disease risk factors and diabetes in Chinese: an approach to the criteria of obesity in the Chinese population. Obes Rev. 2002;3(3):167-72.
27. Jia Z, Zhou Y, Liu X, Wang Y, Zhao X, Liang W, et al. Comparison of different anthropometric measures as predictors of diabetes incidence in a Chinese population. Diabetes Res Clin Pract. 2011;92(2):265-71.

28. Ezeukwu AO, Agwubike EO: Anthropometric measures of adiposity as correlates of atherogenic index of plasma in non-obese sedentary Nigerian males. Libyan J Med. 2014; 9(23798):

29. Ehrampoush E, Arasteh P, Homayounfar R, Cheraghpour M, Alipour M, Naghizadeh MM, et al. New anthropometric indices or old ones: which is the better predictor of body fat? Diabetes Metab Syndr. 2017;11(4):257-63.

30. Wang H, Liu A, Zhao T, Gong X, Pang T, Zhou Y, et al. Comparison of anthropometric indices for predicting the risk of metabolic syndrome and its components in Chinese adults: a prospective, longitudinal study. BMJ Open. 2017;7(9):2017-016062.

31. Kawada T, Andou T, Fukumitsu M. Metabolic syndrome showed significant relationship with carotid atherosclerosis. Heart Vessel. 2016;31(5):664-70.

32. Lemieux I, Almeras N, Mauriege P, Blanchet C, Dewailly E, Bergeron J, et al. Prevalence of 'hypertriglyceridemic waist' in men who participated in the Quebec health survey: association with atherogenic and diabetogenic metabolic risk factors. Can J Cardiol. 2002;18(7):725-32.

33. Blaak E. Gender differences in fat metabolism. Curr Opin Clin Nutr Metab Care. 2001;4(6):499-502.

34. Li S, Ho SC, Sham A. Relationship between menopause status, attitude toward menopause, and quality of life in Chinese midlife women in Hong Kong. Menopause. 2016;23(1):67-73.

35. Dai D, Chang Y, Chen Y, Chen S, Yu S, Guo X, et al.: Visceral Adiposity Index and Lipid Accumulation Product Index: Two Alternate Body Indices to Identify Chronic Kidney Disease among the Rural Population in Northeast China. Int J Environ Res Public Health. 2016; 13(12):

36. Zhang X, Shu XO, Li H, Yang G, Xiang YB, Cai Q, et al. Visceral adiposity and risk of coronary heart disease in relatively lean Chinese adults. Int J Cardiol. 2013;168(3):2141-5.

37. Salazar MR, Carbajal HA, Espeche WG, Aizpurua M, Maciel PM, Reaven GM. Identification of cardiometabolic risk: visceral adiposity index versus triglyceride/HDL cholesterol ratio. Am J Med. 2014;127(2):152-7.

38. Knowles KM, Paiva LL, Sanchez SE, Revilla L, Lopez T, Yasuda MB, et al. Waist circumference, body mass index, and other measures of adiposity in predicting cardiovascular disease risk factors among Peruvian adults. Int J Hypertens. 2011;24(931402):931402.

39. Sasai H, Brychta R, Wood RP, Rothney MP, Zhao X, Skarulis MC, et al. Does visceral fat estimated by dual-energy $\mathrm{X}$-ray absorptiometry independently predict Cardiometabolic risks in adults? J Diabetes Sci Technol. 2015;9(4):917-24.

40. Park HJ, Kim J, Park SE, Park CY, Lee WY, Oh KW, et al. Increased risk of subclinical atherosclerosis associated with high visceral adiposity index in apparently healthy Korean adults: the Kangbuk Samsung health study. Ann Med. 2016;48(6):410-6

41. Moon BS, Park HJ, Lee MK, Jeon WS, Park SE, Park CY, et al. Increased association of coronary artery calcification in apparently healthy Korean adults with hypertriglyceridemic waist phenotype: The Kangbuk Samsung Health Study. Int J Cardiol. 2015;194:78-82.

42. Taverna MJ, Martinez-Larrad MT, Frechtel GD, Serrano-Rios M. Lipid accumulation product: a powerful marker of metabolic syndrome in healthy population. Eur J Endocrinol. 2011;164(4):559-67.

43. loachimescu AG, Brennan DM, Hoar BM, Hoogwerf BJ. The lipid accumulation product and all-cause mortality in patients at high cardiovascular risk: a PreCIS database study. Obesity. 2010;18(9):1836-44.

44. Kato A, Ishida J, Endo Y, Takita T, Furuhashi M, Maruyama Y, et al. Association of abdominal visceral adiposity and thigh sarcopenia with changes of arteriosclerosis in haemodialysis patients. Nephrol Dial Transplant. 2011;26(6):1967-76.

45. Ruscica M, Baragetti A, Catapano AL, Norata GD. Translating the biology of adipokines in atherosclerosis and cardiovascular diseases: gaps and open questions. Nutr Metab Cardiovasc Dis. 2017;27(5):379-95

46. Henrichot E, Juge-Aubry CE, Pernin A, Pache JC, Velebit V, Dayer JM, et al. Production of chemokines by perivascular adipose tissue: a role in the pathogenesis of atherosclerosis? Arterioscler Thromb Vasc Biol. 2005;25(12): 2594-9.

47. Kwok KH, Cheng KK, Hoo RL, Ye D, XU A, Lam KS. Adipose-specific inactivation of JNK alleviates atherosclerosis in apoE-deficient mice. Clin Sci. 2016;130(22):2087-100 